\title{
Morphometrical findings among dysplasias of oral, cervical and bronchial regions
}

\author{
Kayo Kuyama ${ }^{1,2}$, Takashi Matsumoto ${ }^{2}$, Miyuki Morikawa ${ }^{1,2}$, Akira Fukatsu' ${ }^{3}$, Mana Ichimura ${ }^{3}$, \\ Masanobu Wakami ${ }^{4}$, Masahiko Fukumoto ${ }^{3}$, Taku Kato ${ }^{5}$, Hirotsugu Yamamoto ${ }^{1,2}$ \\ ${ }^{1}$ Department of Oral Pathology, Nihon University School of Dentistry at Matsudo, Chiba, Japan \\ ${ }^{2}$ Department of Diagnostic Pathology, Hospital of Nihon University School of Dentistry at Matsudo, Chiba, Japan \\ ${ }^{3}$ Department of Laboratory Medicine for Dentistry, Nihon University School of Dentistry at Matsudo, Chiba, Japan \\ ${ }^{4}$ Department of Crown and Bridge Orosthodontics, Nihon University School of Dentistry at Matsudo, Chiba, Japan \\ ${ }^{5}$ Clinical Laboratory Section, Funabashi Municipal Medical Center, Chiba, Japan \\ Email: kuyama.kayo@nihon-u.ac.jp
}

Received 7 February 2013; revised 25 March 2013; accepted 8 April 2013

Copyright (C) 2013 Kayo Kuyama et al. This is an open access article distributed under the Creative Commons Attribution License, which permits unrestricted use, distribution, and reproduction in any medium, provided the original work is properly cited.

\section{ABSTRACT}

The purpose of this study was to compare among dysplasia of oral, uterine cervix and bronchus. Using a computer cytomorphometry cell measurement program, the study was based on a retrospective review of smear cases diagnosed with dysplasia of oral, bronchial and uterine cervix, from 2002 to 2010. For 50 70 cells from each lesion, nuclear $(N)$ and cytoplasm (C) variables were assessed: area (A), diameter (D), irregularity (I), stain brightness and granularity. NA and ND were highest in OSCC and higher according to dysplastic grading. By contrast, $C A$ and $C D$ were lowest in severe dysplasia. The significant difference of N/C ratio was observed among OSCC to inflammation, mild and moderate dysplasias $(p<0.05)$. The N/C ratios of mild and moderate dysplasias were equal. Brightness and granularity values of OSCC cases were significantly higher than those of another ( $\mathbf{p}<\mathbf{0 . 0 5}$ ). About the difference between mild to moderate dysplasias, it was the easiest to detect of the uterine cervix. All severe dysplasias among the 3 regions were easily identified morphometrically. The deficient in the difference between inflammation to mild dysplasia and mild to moderate dysplasia were obtained in the oral mucosal lesion. The results displayed a significant variation in cytomorphometrical values among the 3 regions. $N / C$ values for uterine cervix and bronchus were well distinguished in comparison with oral dysplasias. Screening of mild and moderate dysplasias requires experience which carries out the comprehensive judgment of the color.

Keywords: Exfoliative Cytology; Dysplasia; Oral
Mucosa; Cervix; Bronchus

\section{INTRODUCTION}

Since direct observation was carried out anatomically, the oral epithelial dysplasia was delayed in the development of cytological diagnosis as compared with another province region. In Japan, although the cytological diagnosis of the oral mucosa has spread through urgency in recent years, there is very little cytoscreener which makes it a specialty. Therefore, cytoscreener applies the diagnostic criteria of cervix and bronchus to the oral mucosa in many cases. However, although 3 regions are covered with the squamous cell, under diagnosis may be drawn when the same cytological criteria are applied. Since it differs also embryologically, although epithelial dysplasias of 3 regions must prepare individual diagnostic criteria, these cytological comparative studies are not developed.

The term "squamous dysplasia" is commonly used among the oral, cervical and bronchial epithelial dysplasias, although the pathological criteria of dividing the grading are different [1]. During embryonic development, the primitive embryonic ectoderm gives origin to the lining epithelium of the palate, gingiva and cheeks while the embryonic endoderm gives origin to the lining epithelium of the trachea and the small air passages. The intraembryonic mesoderm gives origin to the female uterine tube and uterus [2]. The pathological assessment of oral precursor lesions according to WHO classification described the spectrum of dysplasia into mild, moderate and severe categories [3]. Mild dysplasia is the first histological event in the spectrum of these 3 regions. Moderate and severe dysplasias are the subsequent phases 
that precede invasive carcinoma and there is a good correlation between higher grades of dysplasia and increasing risk of cancer, but studies of the correlation between dysplastic grading and malignancy rate are few [4].

Exfoliative cytology is an effective screening method, which had been used by Papanicolaou in the past and between 1941-1942, the technique was put into practical use in gynecology when the cancer cells were demonstrated in vaginal smears $[5,6]$. Hence, the accuracy rate of exfoliative cytology in cervix was high $[7,8]$. At the same time examinations of bronchial secretions have appeared largely in 1943 since Papanicolaou and Traut but the development of pulmonary cytology was marked by the first diagnosis of lung cancer by cytologic examination of sputum in 1887 by Hampeln $[1,9,10]$. It was suggested that sputum biopsy was the most accurate diagnostic procedure [11,12]. Since then, the criteria are almost established for cervical uterine and bronchus. Interest in the exfoliative cytology of the oral cavity dates back as far in 1890 and it was felt that its application to lesions of the oral cavity might be successful $[13,14]$. However, there are few reports about exfoliative cytology for oral lesions and for cytological findings of oral dysplasia and compare with cervix and bronchus [15-17].

Since cytological diagnosis of oral dysplasia has copied the standard of the cervical dysplasia until now, there are no original diagnostic criteria. Attempts have been made to quantify the dysplastic changes using computeraided analysis and Cowpe et al., advocated the idea of using morphometry for enhanced diagnosis of lesions using cytological techniques $[18,19]$. The cell morphometry features were measured from Papanicolaou stained smears and the cytomorphometrical criteria such as nuclear area, cytoplasmic area, and nuclear/cytoplasmic ratio were considered. The study was aimed to determine the morphological features among these 3 regions, in an attempt to establish a better morphological definition and criteria in oral epithelial dysplasia. Thus, similar morphological changes develop in the oral, bronchial and cervical epithelia before invasive carcinoma happens afterwards [20]. Oral dysplasia has many macroscopic findings and it is manifested typically as a white, mixed red or white lesion and sometimes papillary, nodular, pumice, ulcerative or mixed appearance [21]. Its clinical characteristics can be guessed but its severity cannot be accurately predicted macroscopically. The relationship of epithelial dysplasia to the subsequent development of cancer has not been fully clarified [22]. It has been reported that there is a marked variation in oral malignant transformation rates in studies from different areas of the world, ranging from $1.4 \%$ to $36.4 \%$ with observational periods ranging from 1 month to 15 years or more [23,24]. Therefore, it became apparent that there was a need for early diagnosis of malignant lesions using exfolaitive cytology which is the most simple and cost effective method of cancer screening.

In order to establish a realistic evaluation of cytological findings of oral epithelial dysplastic lesions, our present study was undertaken to assess the dysplastic cells microscopically and morphometrically. The comparative study among oral, cervix and bronchial dysplasias was also performed.

\section{MATERIALS AND METHODS}

\subsection{Smear Specimens}

The study was based on a retrospective review of cytological examination of cases diagnosed with dysplasia of oral, bronchial and uterine cervix, from 2002 to 2011. Alloral smears from normal $(\mathrm{n}=20)$, inflammation $(\mathrm{n}=$ $20)$, mild dysplasia $(n=10)$, moderate dysplasia $(n=10)$, severe dysplasia $(\mathrm{n}=10)$, and OSCC (well differentiated type, $n=10$ ) were selected from the archive of Nihon University School of Dentistry at Matsudo. And the cervical $(\mathrm{n}=30)$ and bronchial lesions $(\mathrm{n}=25)$ were obtained from the Funabashi Municipal Medical Centre. The classification of the cases wasmild dysplasia $(\mathrm{n}=$ $10)$, moderate dysplasia $(n=10)$, severe dysplasia $(n=$ $10)$ of the cervix and mild dysplasia $(n=10)$, moderate dysplasia $(n=10)$, severe dysplasia $(n=5)$ of the bronchus. The protocol was approved by the Committee of Studies Involving Human Beings of Nihon University School of Dentistry at Matsudo (E-5.002).

From each oral mucosal and uterine cervical lesion, cells were exfoliated using cytobrush (Medscand Medical, CoperSurgical Company, Berlin, Germany) from mucosal lesions. All smears were performed before biopsy. The bronchial mucosal smears were collected using bronchoscopic brush during bronchoscopy. Informed consent was obtained from all patients before taking the cytological smears. All these patients showed dysplastic or suspicious change under macroscopical findings.

To obtain a smear, the cytobrush was rolled at least 5 times with gentle pressure. The clinician took smears for each patient and gently smeared on labeled glass slides, immediately fixed in $95 \%$ ethyl alcohol, and subsequently stained by the Papanicolaou method and checked by five cytologists for cytological diagnosis and confirmed by six pathologists. The fixation and staining times were standardized between 2 facilities.

\subsection{Cytological Assessment of Smears}

Immunohistochemically, the fascicles of tumor cells were positive for S-100 protein (Figure 1(g)), vimentin (Figure 1(h)) and negative for $\alpha$-actin and GFAP (Figure 1(i)). A few numbers of small neuroid structure and ax- 

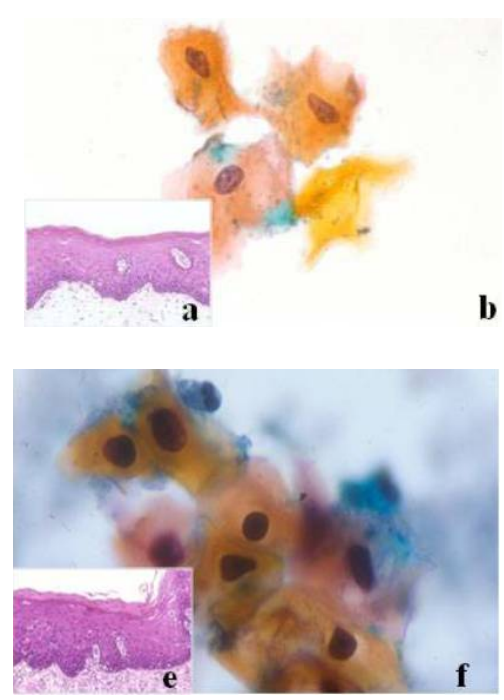

b

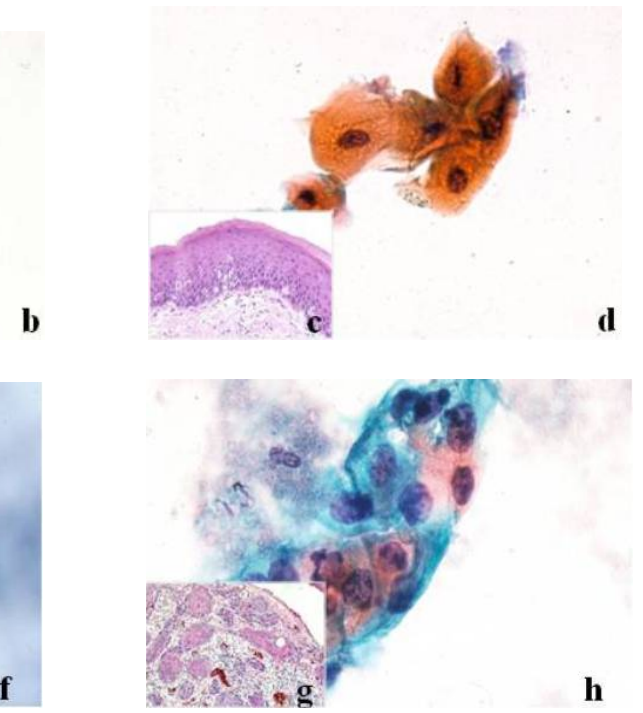

Figure 1. Histopathological and cytological figures of mild dysplasia of oral mucosa. (a) Histopathological finding of leukoplakia shows mild dysplasia with atypical epithelial features confined to the lower third of the epithelium (HE, original magnification $\times 200$ ); (b) Cytological findings show mild dysplastic features: enlarged nuclei with slight hyperchromasia and eosinophilic (orange) cytoplasms. It was diagnosed as mild dysplasia (Pap. $\times 400$ ); (c) Histopathological finding of moderate dysplasia with atypical epithelial features confined to the lower half of the epithelium (HE, original magnification $\times 200$ ); (d) Cytological findings show moderate cytological changes of nuclei (Pap. $\times 400$ ); (e) Histopathological finding of severe dysplasia showing nearly total thickness and alterations extending to upper third of epithelium (HE, original magnification $\times 200$ ); (f) Cytological findings show severe cytological changes of nuclei with hyperchromasia, irregular border and thick keratinized cytoplasm (Pap. $\times$ 400); (g) Histopathological findings of OSCC (HE, original magnification $\times 200$ ); (h) Cytological finding shows the prominent cytological features of OSCC. The nuclei are strongly hyperchromatic, coarse granularity, irregular border with strong eosinophilic and basophilic cytoplasms, and high N/C ratio. The cytoplasmic borders are ill-defined (Pap. ×400).

ons were demonstrated by NSE and NF in this lesion, respectively. These results indicate that the tumor is composed of cells of schwannian differentiation and they also indicate the presence of axons. Therefore, the definitive diagnosis was PEN. There was no relationship with von Recklinghausen's disease, and none of the lesions recurred after excision.

\subsection{Histopathological Assessment}

Tissue specimens from both biopsy and operational materials of the oral mucosal lesions were routinely stained with hematoxylin and eosin (HE) and subjected to histopathological review examination. Histopathologically, all dysplasias were categorized as mild, moderate and severe, based on severity of histomorphological features according to WHO criteria [3]. All subjects the study used, the histopathological and cytological diagnoses were matching, except the bronchial smears were without any histological specimens that consisted exclusively of atypical metaplastic cells from the upper airways where diagnosis was based on the severity of the smears. The assessments of the histological criteria for uterine cervix mucosa were also made by referring to the general rules for clinical studies according to the Japan society for uterine cervical premalignancies and malignancies.

\subsection{Cytomorphometrical Assessment}

On the basis of the cytomorphological characteristics, the frequencies of changes of dysplastic cells were analyzed. In each dysplastic category, an average of between 100 to 150 accurately selected uniform atypical cells was analyzed by using a computer cytomorphometry cell measurement program (Win Roof version 2.5, Mitani Co.) $[18,19,27]$. Only smears with unclamped, monolayered and uniform squamous cells were used for this study. The study also tried to find out the differences between various categories of oral dysplastic cells compared to non-dysplastic and OSCC chromatin and color density in order to better understand the morphometrical parameters of oral dysplasia. 
The mean nuclear and cytoplasmic area and diameter (NA, ND, CA, CD), irregularity (NI, CI), and N/C ratio of all cases were the parameters of interest in this study and their mean values were obtained in micrometers for diameters and the area in square micrometers. Also, the mean nuclear and cytoplasmic color brightness and granularity or degree of chromatin coloring (NB, NG, CB, $\mathrm{CG}$ ) of all keratinizing oral dysplastic categories, normal, inflammatory and OSCC cases and bronchial and cervical dysplasias were also analyzed.

\subsection{Statistical Analysis}

The descriptive analyses of the morphometrical variables of area and diameter were stated with mean value in micrometer. A $t$ test was used to compare the significant difference for NA and ND, CA and CD and NB, NG, CB, $\mathrm{CG}$ in all groups using statistical program (SPSS 11.OJ). A p value of less than 0.05 was considered significant.

\section{RESULTS}

\subsection{Histopathological and Cytological Findings}

As for the oral smears, mix light green and yellow cells with increased nuclei but with no dysplastic characteristics and many inflammatory cells were shown in the inflammatory lesion. Mild dysplasia demonstrated basal cell hyperplasia with relatively mild cytological changes confined to lower third of epithelium (Figure 1(a)). Cytologically, itpresented minor degree of nuclei atypical changes such as slight hyperchromatism, but uniform and coarsely granular and slight irregularity in shape (Figure 1(b)). Moderate dysplasia demonstrated cytological atypia extending to mid-third of epithelium (Figure 1(c)). It showed more marked nuclear atypical changes compared with mild dysplasia, cytologically (Figure 1(d)). Severe dysplasia demonstrated dysplastic change into upper third of epithelium with prominent cytological change with the most marked nuclear abnormalities (Figures 1(e) and (f)). OSCC showed the characteristics of carcinoma such as irregular shape, hyperchromatic nuclei and high N/C ratio with necrotic background (Figures $\mathbf{1 ( g )}$ and (h)).

In uterine cervix, mild and moderate dysplasias were dominated by superficial and intermediate green and yellow squamous cells with a subdominant population of parabasal cells (Figures 2(a)-(d)). In severe dysplasia the parabasal cells were remarkably observed than intermediate cells (Figures 2(e) and (f)). The presence of koilocytic atypia of superficial and intermediate cells was common features unlike the oral squamous cells. Nuclear cleavage and the circular wrinkles in cytoplasm were also features in uterine cervical cells but not in oral squamous cells. Many red and green squamous metaplastic cells with nuclear atypia were selected from the mild, moderate and severe bronchial smears (Figures 3(a)-(c)). The size of the metaplastic cells was smaller compared with the oral and uterine cervix squamous cells.

\subsection{Morphometrical Findings of Oral Lesions}

Table 1 shows the morphometrical findings of oral lesions. The mean NA and ND values showed an increase from normalthrough lesions with inflammation and reduced through mild and moderate dysplasias, and increased in severe and OSCC. Among dysplasias, mild and moderate NA values were close to normal. Regarding the CA mean values of severe and OSCC compared with that of normal: 7 and 14 bigger than severe dysplasia and OSCC, respectively. The "irregularity": NI and CI means values showed no differences in oral lesions. The difference between the NA of normal compared with dysplasias and OSCC were significant $(\mathrm{p}<0.05)$. The mean N/C ratio was significant from normal, mild and moderate dysplasias to OSCC $(\mathrm{p}<0.05)$.
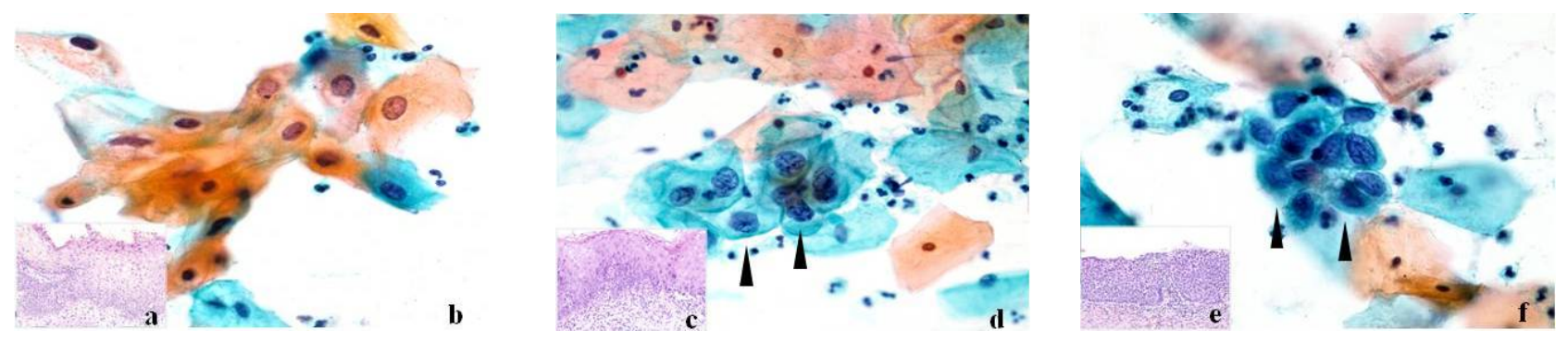

Figure 2. Histopathological and cytological figures of mild dysplasia of uterine cervix. (a) Histopatholgical findings of mild dysplasia of uterine cervix (HE, original magnification $\times 200$ ); (b) Cytological findings show mild dysplastic superficial and intermediate green and red squamous cells with koilocytic atypia (Pap. $\times 400$ ); (c) Histopathological findings of moderate dysplasia of uterine cervix (HE, original magnification $\times 200$ ); (d) Cytological finding of moderate dysplasia of uterine cervix showing superficial and intermediate green and red squamous cells and a few parabasal cells with koilocytic atypia (arrowheads, Pap. $\times 400$ ); (e) Histopathological findings of severe dysplasia of uterine cervix (HE, original magnification $\times 200$ ); (f) Cytological finding of severe dysplasia of uterine cervix showing superficial and intermediate green and red squamous cells and many parabasal cells with koilocytic atypia (arrowheads, Pap. $\times 400$ ). 

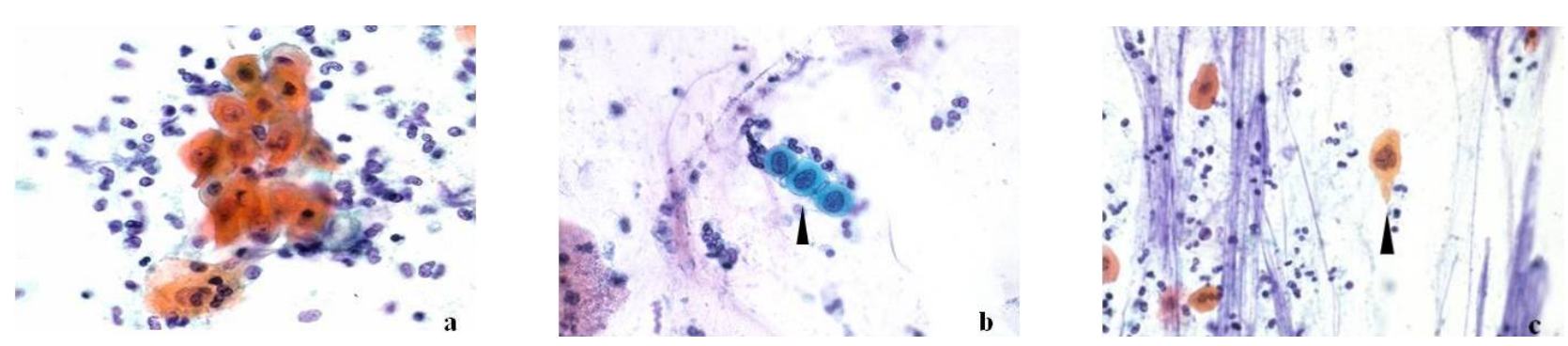

Figure 3. Histopathological and cytological figures of mild dysplasia of bronchus. (a) Cytological findings of mild bronchial dysplasia with yellow metaplastic squamous cells. Many inflammatory cells are seen (Pap. $\times 600$ ); (b) Cytological findings of moderate bronchial dysplasia with blue metaplastic squamous cells and many inflammatory cells (arrowhead, Pap. $\times 600$ ); (c) Cytological findings of severe bronchial dysplasia showing a yellow metaplastic multinucleated squamous cell with many inflammatory cells (arrowhead, Pap. $\times 600)$.

Table 1. The mean cytomorphological values between lesions of the oral mucosa.

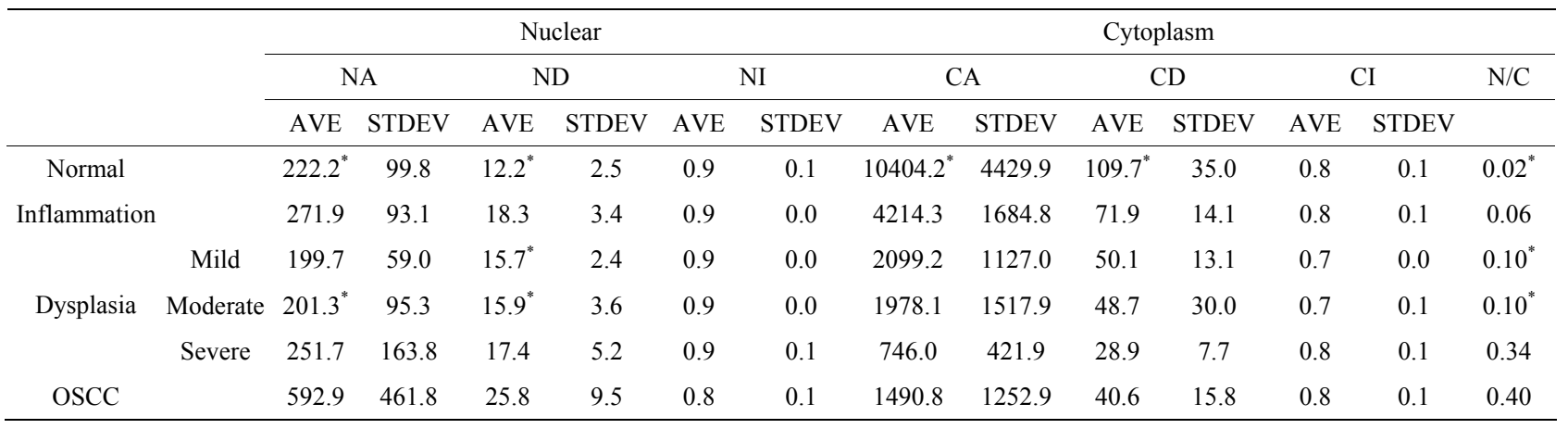

AVE: average; STDEV: standard deviation; NA: nuclear area $(\mu \mathrm{m})$, ND: nuclear diameter $(\mu \mathrm{m})$; CA: cytoplasmic area $(\mu \mathrm{m}), \mathrm{CD}$ : cytoplasmic diameter $(\mu \mathrm{m})$; N/C: nuclear cytoplasm ratio; Significant differences $\left({ }^{*}<0.05\right)$ was observed compared to OSCC.

\subsection{Morphometrical Findings of Oral Lesions Nuclei and Cytoplasm Color Saturation}

Table 2 shows the color saturation mean values for NB, NG, CB and CG. As for NB, there was close differences among normal, inflammation and mild dysplasia, and also between severe dysplasia and OSCC. However, NB and NG mean values showed an increase in mild dysplasia and further increased in moderate and severe dysplasias. CB and CG mean values showed the same trend from normal to inflammation, dysplasias and OSCC. Regarding the OSCC cases, the mean NB and NG values were significantly higher than those of dysplasias, inflammation and normal $(\mathrm{p}<0.05)$.

\subsection{Morphometrical Findings among Oral, Cervical and Bronchial Dysplastic Cases}

Table 3 shows the comparative cytomorphometrical results among 3 regions. The NA and ND values showed the same tendency of a decline from mild to moderate dysplasias, and severe dysplasia was the most highest. Among the 3 regions, uterine cervix had the highest NA and ND mean values. CA mean values were also highest in uterine cervix while $\mathrm{CD}$ was increased from mild to moderate dysplasias and declined in the severe ones. NI and CI did not show any differences. Regarding the mean
$\mathrm{N} / \mathrm{C}$ ratio among the 3 regions, it increased significantly with dysplastic grading and highest in uterine cervix $(\mathrm{p}<$ 0.05 ). From mild to moderate dysplasias, the uterine cervix showed the greatest differences in comparison with oral dysplasias. All severe dysplasias among the 3 region were easily identified morphometrically.

\section{DISCUSSION}

Although early diagnosis of OSCC plays the most important role for the increase of the survival rate of patients, the 5-year survival rate has remained under 50\% [28]. But, the early diagnosis of OSCC is difficult, due to the asymptomatic nature and benign appearance of these lesions on initial presentation. The major advantage of exfoliative cytology is the noninvasive character of the technique, which allows a simple and pain-free collection of intact cells from different layers in the epithelium for microscopical examination and quantitative evaluation [8]. By increasing detection of premalignancies and early invasive disease, the use of exfoliative cytology by Papanicolaou has decreased both the incidence and mortality of cervical cancer $[29,30]$. On the contrary, sensitivity and specificity of cytological diagnosis for oral mucosal diseases showed 55\% and $100 \%$, respectively [31]. Since the subject which decreases false-positive 
Table 2. The mean cytomorphological values between lesions of the oral mucosa.

\begin{tabular}{cccccc}
\hline & & \multicolumn{3}{c}{ Cytoplasm } \\
\cline { 3 - 6 } & & Brightness & Granularity & Brightness & Granularity \\
\hline Normal & $96.3^{*}$ & $80.2^{*}$ & $96.3^{*}$ & $78.3^{*}$ \\
Inflammation & & $99.1^{*}$ & 116.2 & 146.2 & 123.8 \\
& Mild & 105.2 & 174.7 & 150.5 & 173.4 \\
Dysplasia & Moderate & 130.3 & 179.9 & 158.8 & 189.5 \\
& Severe & 140.6 & 185.6 & 160.7 & 200.3 \\
OSCC & & 155.5 & 195.5 & 175.9 & 230.9 \\
\hline
\end{tabular}

Significant differences $\left({ }^{*}<0.05\right)$ was observed compared to OSCC.

Table 3. The mean cytomorphometrical values among regions and within each category of dyplasia $(\mu \mathrm{m})$.

\begin{tabular}{|c|c|c|c|c|c|c|c|c|c|c|c|c|c|c|}
\hline & & \multicolumn{6}{|c|}{ Nuclear } & \multicolumn{6}{|c|}{ Cytoplasm } & \multirow{3}{*}{$\mathrm{N} / \mathrm{C}$} \\
\hline & & \multicolumn{2}{|c|}{ NA } & \multicolumn{2}{|c|}{ ND } & \multicolumn{2}{|c|}{ NI } & \multicolumn{2}{|c|}{$\mathrm{CA}$} & \multicolumn{2}{|c|}{$\mathrm{CD}$} & \multicolumn{2}{|c|}{ CI } & \\
\hline & & AVE & STDEV & AVE & STDEV & AVE & STDEV & AVE & STDEV & AVE & STDEV & AVE & STDEV & \\
\hline \multirow{3}{*}{$\begin{array}{c}\text { Oral } \\
\text { Dysplasia }\end{array}$} & Mild & 199.7 & 59.0 & 15.5 & 2.4 & 0.9 & 0.0 & 2099.2 & 1127.0 & 50.1 & 13.1 & 0.7 & 0.0 & $0.10^{*}$ \\
\hline & Moderate & 199.9 & 95.3 & 15.7 & 3.6 & 0.9 & 0.0 & 2941.1 & 1517.9 & 58.7 & 30.0 & 0.7 & 0.1 & 0.10 \\
\hline & Severe & 251.7 & 163.8 & 17.4 & 5.2 & 0.9 & 0.1 & 746.0 & 421.9 & 28.9 & 7.7 & 0.8 & 0.1 & 0.34 \\
\hline \multirow{3}{*}{$\begin{array}{c}\text { Cervical } \\
\text { Dysplasia }\end{array}$} & Mild & 385.4 & 272.2 & 21.0 & 7.1 & 0.9 & 0.1 & 2850.3 & 2672.6 & 54.9 & 25.1 & 0.8 & 0.1 & $0.14^{*}$ \\
\hline & Moderate & 367.1 & 186.4 & 20.7 & 6.3 & 0.8 & 0.1 & 1857.7 & 1241.5 & 70.3 & 20.9 & 0.7 & 0.1 & 0.20 \\
\hline & Severe & 423.6 & 221.1 & 22.5 & 5.6 & 0.9 & 0.1 & 1029.9 & 597.8 & 34.9 & 9.8 & 0.8 & 0.1 & 0.41 \\
\hline \multirow{3}{*}{$\begin{array}{l}\text { Bronchial } \\
\text { Dysplasia }\end{array}$} & Mild & 149.3 & 60.1 & 13.8 & 4.2 & 0.9 & 0.1 & 768.6 & 356.8 & 30.2 & 7.3 & 0.8 & 0.1 & $0.19^{*}$ \\
\hline & Moderate & 141.5 & 88.1 & 12.8 & 3.8 & 0.9 & 0.0 & 680.4 & 279.9 & 28.8 & 6.2 & 0.9 & 0.1 & 0.21 \\
\hline & Severe & 160.9 & 41.7 & 15.5 & 1.5 & 0.9 & 0.1 & 633.6 & 194.8 & 27.7 & 4.2 & 0.9 & 0.1 & 0.24 \\
\hline
\end{tabular}

AVE: average; STDEV: standard deviation; NA: nuclear area $(\mu \mathrm{m})$, ND: nuclear diameter $(\mu \mathrm{m})$; CA: cytoplasmic area $(\mu \mathrm{m}), \mathrm{CD}$ : cytoplasmic diameter $(\mu \mathrm{m})$; N/C: nuclear cytoplasm ratio; Significant differences $\left({ }^{*}<0.05\right)$ was observed compared to severe dysplasia.

rate remains, its diagnostic value via conventional cytology is seen controversially. Then, the purpose of this study detected the cytological characteristics which lead false-negative, and it aimed at accumulating diagnostic criteria for cytoscreener or dentist peculiar to the oral epithelial dysplasia.

The transformation rate of oral premalignancies is about $14 \%$ over a period of 20 years has been reported [32]. The representative oral premalignant lesion was leukoplakia with thick keratinizing superficial layer and epithelial dysplasia. It was graded as mild, moderate and severe dysplasia according to the WHO criteria [3]. Microscopically, a small number of dysplastic cells with keratin debris and the atypia showed scare pyknotic nuclei. As for N/C ratio, mild and moderate were similar to inflammatory lesions. The cytology of severe and OSCC was comparatively detectable. More cytological observation should be needed and since the use of this technique may improve the diagnostic reliability of exfoliative cytology in the management of oral premalignancies.

Regarding the cervical uterine and bronchial lesions, many dysplastic cells were observed with obvious atypia and many reports had accumulated the cytological find- ings; cellular atypia and N/C ratios were increasing with dysplastic grading $[5-7,12,16]$. Therefore, the criteria of screening cytology for these two regions were almost standardized. As for oral, the study showed that decrease in NA among dysplasias reflected the ability of morphometry to detect alterations in nuclei contents constitute early events in the carcinogenic process [33]. Hence, the differences between mild and moderate were small and between mild and severe ones were significant. The study also found out that severe dysplasia and OSCC displayed CA reduction compared with cancer-free cases and early stages of dysplasias. Therefore, the increase in NA and decrease in CA are useful early indicators of malignancy. The NI and CI did not show any significance and the morphometrical analysis cannot distinguish between the cases but the morphometrical color analysis was shown to distinguish among the oral lesions and dysplasias. This result also showed that small differences on NA, ND and N/C between inflammatory lesions and mild dysplasia in comparison with uterine cervix and bronchial dysplasias. Similar value of NA and $\mathrm{N} / \mathrm{C}$ of oral inflammation versus mild dysplasia and mild dysplasia versus moderate dysplasia were led to overlook 
the diagnoses.

There is a challenge in the recognition of the earliest manifestations of dysplasia. It was suggested that moderate intraepithelial dysplasias were risky epithelium while Bethesda system defined moderate intraepithelial dysplasias as high grade cervical squamous intraepithelial neoplasia (CIN2) [34,35]. Therefore, the diagnosis of moderate dysplasias is important because moderate dysplasias are regarded as intraepithelial tumor. Cell shape, size, irregularity and differences between mild and moderate were small. However, the study revealed that the chromatin color distribution could distinguish between mild from moderate and severe oral dysplasias. The study suggested that differences in nuclear staining, especially granularity of chromatin; color estimation of cytoplasm and nuclear, could be used to differentiate between normal, dysplastic and malignant regions. Similar problems were encountered when conventional screening of the smears. It is important, however, to note in this regard that the site would be biopsied, especially that early detection is vital to improve prognosis.

The study also related the differences in the mean cytomorphometrical values among the 3 regions. Both mild to severe dysplasias of both uterine cervix and bronchus showed a remarkable atypical change accompany with dysplastic change while the oral ones were not, suggesting a great consideration regarding oral dysplasias, especially mild and moderate ones. It was found that the risk of progression from mild to severe dysplasia or OSCC was only $1 \%$ per year but the risk of progression from moderate dysplasia was $16 \%$ within two years and $25 \%$ within 5 years [36]. Therefore, moderate category may represent a clinically useful distinction.

On the basis of our results, the study successfully applied the cytomorphometrical parameters of nuclear and cell sizes, configuration, nuclear to cytoplasmic ratio (N/C) and color density and can be evaluated collectively in order to aid in the diagnosis of dysplasia and malignancy from cytological cells. Of these parameters, the nuclear and cytoplasmic areas (NA,CA), and N/C ratios have been shown to be significant in the diagnosis. In the oral region, mild and moderate dysplasias, the $\mathrm{N} / \mathrm{C}$ ratio was hard to detect compared with uterine cervix and bronchial dysplasias. More cytological study of oral dysplastic lesion, particularly chromatin distribution in early stage of dysplasia should accumulate and led to improvement of accuracy.

\section{REFERENCES}

[1] Kuffer, R. and Lombardi, T. (2002) Premalignant lesions of the oral mucosa. A discussion about the place of oral intraepithelial neoplasia (OIN). Oral Oncology, 38, 125130. doi:10.1016/S1368-8375(01)00050-1
[2] Prelle, K., Zink, N. and Wolf, E. (2002) Pluripotent stem cells-model of embryonic development, tools for gene targeting and basis of cell therapy. Anatomia, Histologia, Embryologia, 31, 169-186. doi:10.1046/j.1439-0264.2002.00388.x

[3] Barnes, L., Eveson, J.W., Reichart, P., et al. (2005) Head and neck tumors: epithelial precursor lesions. WHO-classification of tumors. IARC Press, Lyon.

[4] Sudbø, J., Bryne, M., Johannessen, A.C., et al. (2007) Notice of retraction: Comparison of histological grading and large-scale genomic status (DNA ploidy) as prognostic tools in oral dysplasia. Journal of Pathology, 194, 303-310.

doi:10.1002/1096-9896(200107)194:3<303::AID-PATH8 79>3.0.CO;2-Q

[5] Papanicolaou, G.N. and Traut, H.F. (1941) The diagnostic value of vaginal smears in carcinoma of the uterus. American Journal of Obstetrics \& Gynecology, 42, 193206.

[6] Papanicolau, G.N. and Traut, H.F. (1943) Diagnosis of uterine cancer by the vaginal smear. The Commonwealth Fund, New York.

[7] Mayorga, M., Acebo, E. and Val-Bernal, J. (1998) Palisaded encapsulated neuroma of the nasal fossa. Otolaryngology-Head and Neck Surgery, 19, 141-143. doi:10.1016/S0194-5998(98)70196-2

[8] Saslow, D., Runowicz, C.D., Solomon, D., et al. (2002) American cancer society guideline for the early detection of cervical neoplasia and cancer. Cancer Journal for Clinicians, 52, 342-362.

[9] Takac, I., Ursic-Vrscaj, M., Repse-Fokter, A., et al. (2008) Clinicopathological characteristics of cervical cancer between 2003 and 2005, after the introduction of a national cancer screening program in Slovenia. European Journal of Obstetrics \& Gynecology and Reproductive Biology, 140, 82-89. doi:10.1016/j.ejogrb.2008.02.019

[10] Cowar, F.J.S. (1943) Carcinoma of the lung: The value of sputum examination in diagnosis. British Journal of Surgery, 30, 193-196. doi:10.1002/bjs.18003011904

[11] Hampeln, P. (1887) Uber einen fall von primaren lungen pleura-carcinoma. St Petersburg Med Wochenschr, 4, 137-139.

[12] Davies, D.F. (1966) A review of detection methods for the early diagnosis of lung cancer. Journal of Chronic Diseases, 19, 819-845. doi:10.1016/0021-9681(66)90002-6

[13] Lam, B., Lam, S.Y., Wong, M.P., et al. (2008) Sputum cytology examination followed autofluorescence bronchoscopy: A practical way of identifying early stage lung cancer in central airway. Lung Cancer, 64, 289-294. doi:10.1016/j.lungcan.2008.09.016

[14] Montgomery, P.W. (1951) A study of exfoliative cytology of normal human oral mucosa. Journal of Dental Research, 30, 12-18. doi: $10.1177 / 00220345510300010501$

[15] Sandler, H.C. and Stahl, S.S. (1958) Exfoliative cytology as a diagnostic aid in the detection of oral neoplasms. Journal of Oral Surgery, 16, 414-419. 
[16] Abdel-Salam, M., Mayall, B.H., Hansen, L.S., et al. (1987) Nuclear DNA analysis of oral hyperplasia and dysplasia using image cytometry. Journal of Oral Pathology \& Medicine, 16, 431-435. doi:10.1111/j.1600-0714.1987.tb00713.x

[17] Kashyap, V., Das, D.K. and Luthra, U.K. (1990) Microphotometric nuclear DNA analysis in cervical dysplasia of the uterine cervix: Its relation to the progression to malignancy and regression to normalcy. Neoplasm, 37, 497501.

[18] Greenberg, S.D. (1983) Recent advances in pulmonary cytopathology. Human Pathology, 14, 901-912. doi:10.1016/S0046-8177(83)80165-8

[19] Cowpe, J.G., Longmore, R.B. and Green, M.W. (1985) Quantitative exfoliative cytology of normal oral squames: An age, site and sex related survey. Journal of the Royal Society of Medicine, 78, 995-1004.

[20] Ogden, G.R. and Cowpe, J.G. (1989) Quantitative cytophotometric analysis of an aid to the detection of recurrent oral cancer. British Journal of Oral and Maxillofacial Surgery, 27, 224-228. doi:10.1016/0266-4356(89)90149-6

[21] Rubio, C.A. (1981) Letter to the editors-Cellular changes preceding slight dysplasia of the uterine cervix. Acta Cytologica, 25, 193-195.

[22] Shaklar, G., Meyer, I., Cataldo, E., et al. (1968) Correlated study of oral cytology and histopathology-report on 2,052 oral lesions. Oral Surgery, Oral Medicine, Oral Pathology, 25, 61-70. doi:10.1016/0030-4220(68)90200-4

[23] Abbey, L.M., Kaugars, G.E. and Gunsolley, J.C. (1995) Intraexaminer and interexaminer reliability in the diagnosis of oral epithelial dysplasia. Oral Surgery, Oral Medicine, Oral Pathology, Oral Radiology, and Endodontology, 80, 188-191. doi:10.1016/S1079-2104(05)80201-X

[24] Chandler, J.R. (1966) The nonvalue of oral cytology. Archives of Otolaryngology, 84, 527-533. doi:10.1001/archotol.1966.00760030529013

[25] Japan Society of Obstetrics and Gynecology, The Japanese Society of Pathology, and Japan Radiological Society (1997) The general rules for clinical and pathological management of uterine cervical cancer. 2nd Edition, Kanehara, Tokyo.
[26] The Japan Lung Cancer Society (2003) General rule for clinical and pathological record of lung cancer. 6th Edition, Kanehara, Tokyo.

[27] Brickley, M.R., Cowpe, J.G. and Shepherd, J.P. (1996) Performance of a computer simulated neural network trained to categorise normal, premalignant and malignant oral smears. Journal of Oral Pathology \& Medicine, 25, 424-428. doi:10.1111/j.1600-0714.1996.tb00291.x

[28] Cenci, M., Mancini, R., Nofroni, I., et al. (2000) Endocervical atypical glandular cells of undetermined significance: Morphometrical and cytologic characterization of cases that cannot rule out adenocarcinoma in situ. Acta Cytologica, 44, 319-326. doi:10.1159/000328472

[29] Remmerbach, T.W., Weidenbach H., Pomjanski N., et al. (2001) Cytologic and DNA-cytometric early diagnosis of oral cancer. Analytical Cellular Pathology, 22, 211-221.

[30] Ito, E. and Kudo, R. (1982) Scanning electron microscopy of normal cells, dyskaryotic cells and malignant cells exfoliated from the uterine cervix. Acta Cytologica, 26, 457-465.

[31] White, F.H., Jin, Y. and Yang, L. (1997) An evaluation of the role of nuclear cytoplasmic ratios and nuclear volume densities as diagnostic indicators in met plastic, dysplastic and neoplastic lesions of the human cheek. Histology and Histopathology, 12, 69-77.

[32] Kämmerer, P.W., Koch, F.P., Santoro, M., Babaryka, G., Biesterfeld, S., Brieger., J. and Kunkel, M. (2013) Prospective, blinded comparison of cytology and DNA-image cytometry of brush biopsies for early detection of oral malignancy. Oral Oncology, in Press.

[33] Rich, A.M. and Reade, P.C. (1996) Nuclear morphometry in experimental oral mucosal carconogenesis. European Journal of Cancer Part B: Oral Oncology, 32, 169-175. doi:10.1016/0964-1955(95)00086-0

[34] Kurman, R.J., Solomon, D., Nayar, R., et al. (2004) The Bethesda system for reporting cervical cytology: Definitions, criteria, and explanatory notes. Springer, New York.

[35] Tucker, J.H., Cowpe, J.G. and Ogden, G.R. (1994) Nuclear DNA content and morphometrical characteristics of normal, premalignant and malignant oral smears. Analytical Cellular Pathology, 6, 117-128.

[36] Tavassoli, F.A. and Devilee, P. (2003) WHO classification of tumours: Pathology and genetics of tumours of the breast and female genital organs. IARC Press, Lyon. 